\title{
Using Calculus to Model the Growth of L. Plantarum Bacteria
}

\author{
Erin Carey \\ University of South Florida
}

\author{
Advisors: \\ Arcadii Grinshpan, Mathematics and Statistics \\ Scott Campbell, Chemical \& Biomedical Engineering \\ Problem Suggested By: Scott Campbell
}

Follow this and additional works at: https://digitalcommons.usf.edu/ujmm

Part of the Mathematics Commons

UJMM is an open access journal, free to authors and readers, and relies on your support:

Donate Now

\section{Recommended Citation}

Carey, Erin (2009) "Using Calculus to Model the Growth of L. Plantarum Bacteria," Undergraduate Journal of Mathematical Modeling: One + Two: Vol. 1: Iss. 2, Article 2.

DOI: http://dx.doi.org/10.5038/2326-3652.1.2.2

Available at: https://digitalcommons.usf.edu/ujmm/vol1/iss $2 / 2$ 


\title{
Using Calculus to Model the Growth of L. Plantarum Bacteria
}

\begin{abstract}
Experimental data for the growth of Lactobacillus plantarum bacteria have been obtained over time, creating the need for mathematical means to model this data. We use the Gompertz model because it is a sigmoid function for a time series, where growth is slowest at the start and end of a time period. The Gompertz model is especially useful because it defines specific parameters that characterize the Sshaped curve. In addition, the Gompertz model uses relative growth, which is the logarithm of the given population compared to the initial population. This reflects the fact that bacteria grow exponentially. The important parameters that were found were the lag time and the asymptote.
\end{abstract}

\section{Keywords}

Lactobacillus plantarum, Gompertz mode, Asymptote

\section{Creative Commons License}

\section{(c) (1) 99}

This work is licensed under a Creative Commons Attribution-Noncommercial-Share Alike 4.0 License. 


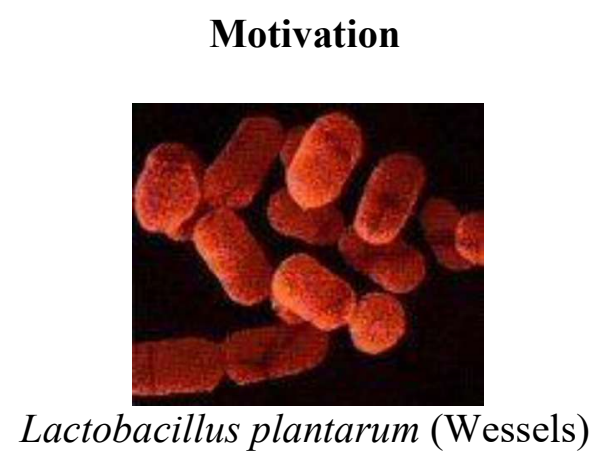

Lactobacillus plantarum bacteria are aerotolerant bacteria that are used in silage, fermented food products and therapeutics. The high levels of this organism in human foods also make it an ideal candidate for the development of probiotics. Furthermore, this bacteria has been shown to reduce Irritable Bowel Syndrome in humans because of its ability to produce antimicrobial substances that help it survive in the gastrointestinal tract of humans (Niedzielin 1144). Thus, the bacteria have the potential to strongly impact the health of both humans and farm animals through the use of food biotechnology.

"Since bacteria reproduce exponentially, it is often useful to plot the logarithm of the relative population size $\left(y=\ln N / N_{0}\right)$ against time. The three phases of the growth curve can be described by three parameters: The maximum specific growth rate is defined as the slope of the tangent at the inflection point, the lag time is defined as the $\mathrm{x}$ axis intercept of this tangent, and the asymptote $\left(A=\lim _{\mathrm{t} \rightarrow \infty} \ln \left(N / N_{0}\right)\right)$ is the maximal value reached" (Zwietering 1876). This theory exemplifies the close relationship between math and science. The ability to model the growth of Lactobacillus plantarum using calculus provides a significant foundation for the development of future products that improve health through the use of this bacteria.

\section{Problem Statement}

\section{Growth curve of $L$ plantarum bacteria}

The typical growth curve for bacteria is $\mathrm{S}$-shaped as shown in the figure below. $N / N_{0}$ is the ratio of number of bacteria, $N$, to the initial population, $N_{0}$, and $t$ is the time. The curve shows an initial lag, followed by exponential growth and then a leveling off. 


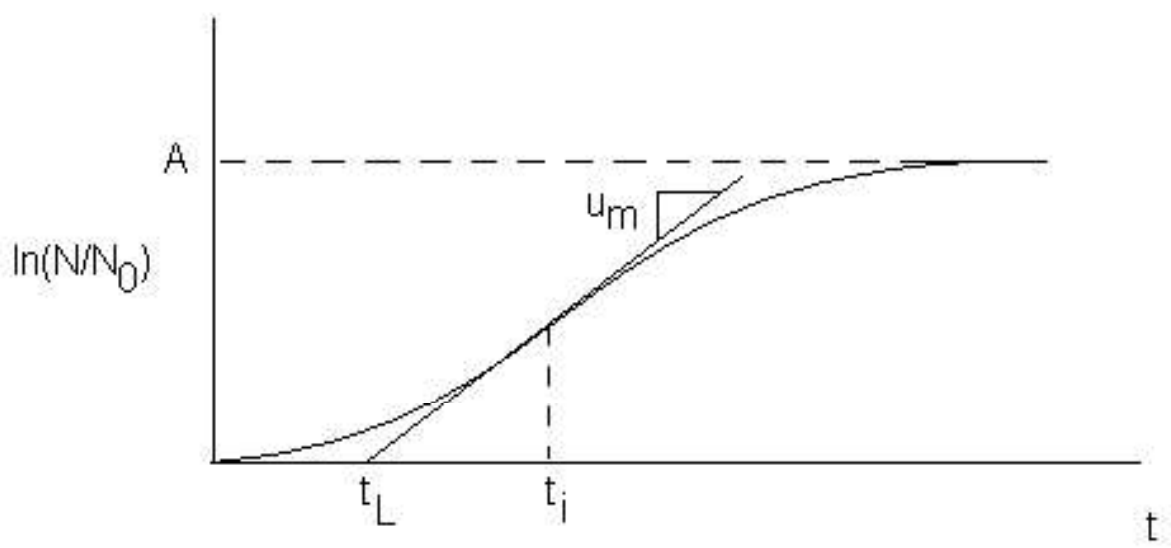

There are several parameters that are useful in characterizing the growth curve. One is the asymptote $A$, which represents the ultimate value of $\ln N / N_{0}$. Another is the lag time $t_{L}$. The lag time can be found as follows:

(1) Obtain the time of the inflection point $t_{i}$.

(2) Find the slope $\mu_{m}$ of the tangent line at the inflection point. This represents the maximum growth rate.

(3) The lag time $t_{L}$ is obtained by finding the $\mathrm{x}$-axis intercept of this tangent line.

A number of models have been used to represent the growth curve. One is the Gompertz model:

$$
\ln \frac{N}{N_{0}}=a e^{-e^{(b-c t)}},
$$

where $a, b$ and $c$ are constants, which are obtained by fitting experimental data. We will use this model here. The table below provides experimental data for the growth rate of an aerotolerant bacteria, $L$ plantarum, at $41.5^{\circ} \mathrm{C}$. Our goal is to obtain the asymptote $A$ and the lag time $t_{L}$. A reasonable approach is as follows:

(a) Fit the Gompertz equation to the data to obtain $a, b$ and $c$.

(b) Show how the parameters $A$ and $t_{L}$ relate to $a, b$ and $c$.

(c) Obtain numerical values of $A$ and $t_{L}$ from step (b). 
Growth data for $L$ plantarum at $41.5^{\circ} \mathrm{C}$

\begin{tabular}{|c|c|}
\hline $\boldsymbol{t}(\mathbf{h r})$ & $\ln \boldsymbol{N} / \boldsymbol{N}_{\mathbf{0}}$ \\
\hline 2 & 0.2 \\
3 & 0.5 \\
4 & 1.1 \\
5 & 1.8 \\
6 & 2.5 \\
8 & 4.0 \\
12 & 6.4 \\
13 & 6.6 \\
\hline
\end{tabular}

\section{Mathematical Description and Solution Approach}

We begin by finding the constants in the Gompertz model that best fit the given data. The Gompertz model is given by

$$
f(t)=a e^{-e^{(b-c t)}}
$$

To determine the asymptote, we take the limit as $t$ approaches infinity:

$$
\text { Asymtope }=\lim _{t \rightarrow \infty} f(t)=\lim _{t \rightarrow \infty} a e^{-e^{(b-c t)}} .
$$

Lag time is obtained by taking the first and second derivatives:

$$
\begin{gathered}
f^{\prime}(t)=a e^{-e^{(b-c t)}} \cdot c e^{(b-c t)}=a c e^{(b-c t)-e^{(b-c t)}} \\
f^{\prime \prime}(t)=a c^{2} e^{(b-c t)-e^{(b-c t)}}\left(e^{(b-c t)}-1\right) .
\end{gathered}
$$

To find the point of inflection, set the second derivative equal to zero. The $\mathrm{x}$-axis intercept of the tangent line gives the lag time.

\section{Discussion}

The first step to finding the growth curve was to find the constants in the Gompertz model that best fit the curve given by the above data. Microsoft Excel was used by inputting the given data and a guess was made as to the three constants. The square in the error of the guesses was calculated. The solver application was then used by manipulating the constants until the sum of the squared errors (sos) was as small as possible. The table below tabulates the results of this procedure. The first two columns give the experimental data; the third column gives the value of $\ln \left(N / N_{0}\right)$ as determined by the Gompertz model 
with constants $a, b$, and $c$ given at the bottom of the table. The final column gives the squared difference. The row with "sos" gives the sum of the square errors. A graph of the curve with the constants given in the table is also shown, along with the given experimental data.

\begin{tabular}{|c|c|c|c|}
\hline$t$ & $\ln \left(N / N_{0}\right)$ & Calc & sqerror \\
\hline 2 & 0.2 & 0.24169 & 0.001738 \\
\hline 3 & 0.5 & 0.562623 & 0.003922 \\
\hline 4 & 1.1 & 1.066686 & 0.00111 \\
\hline 5 & 1.8 & 1.731284 & 0.004722 \\
\hline 6 & 2.5 & 2.498084 & 3.67E-06 \\
\hline 8 & 4 & 4.068512 & 0.004694 \\
\hline 12 & 6.4 & 6.316004 & 0.007055 \\
\hline \multirow[t]{2}{*}{13} & 6.6 & 6.654936 & 0.003018 \\
\hline & & sos & 0.026262 \\
\hline a & 7.832422 & & \\
\hline b & 1.803128 & & \\
\hline $\mathrm{c}$ & 0.278282 & & \\
\hline
\end{tabular}

$$
f(x)=7.8324 \cdot \exp \left\{-e^{(1.8031-0.27828 t)}\right\}
$$

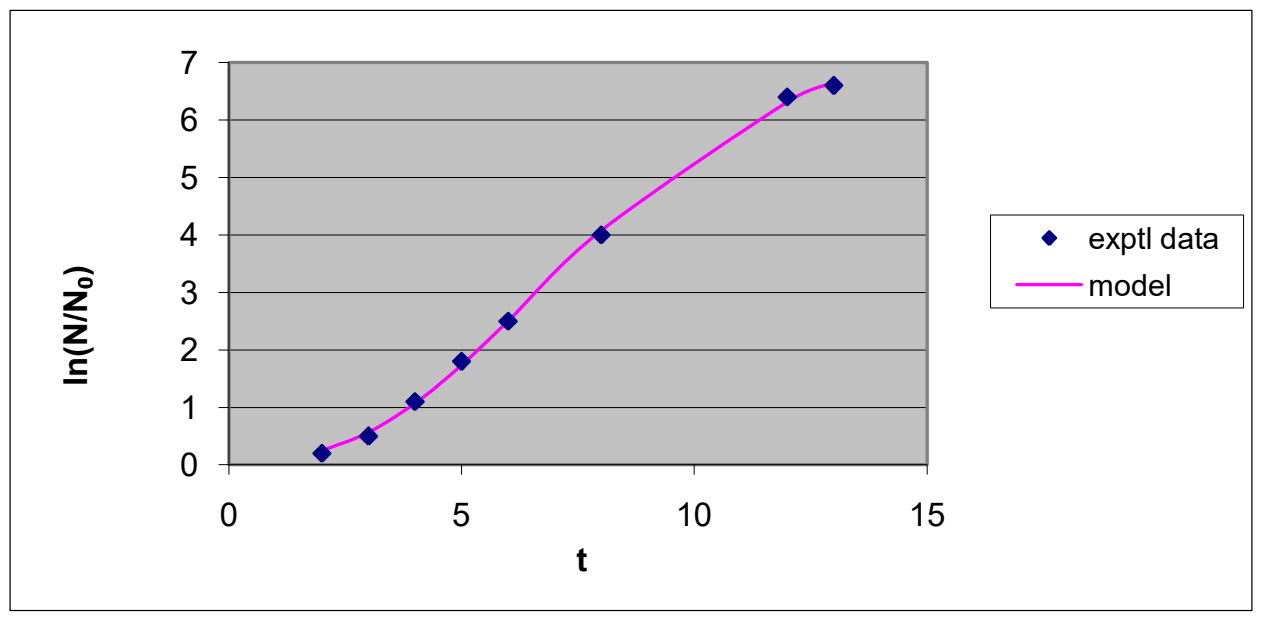

The next step in analyzing the growth curve was to find the parameters indicated above, the asymptote $A$ and the lag time $t_{L}$.

The horizontal asymptote can be found by determining the limit of the given function as $t$ approaches infinity. (See Figure 1 in the appendix.)

$$
A=\lim _{t \rightarrow \infty} 7.8324 \exp \left(-e^{(1.8031-0.27828 t)}\right)=7.8324 .
$$

It was also necessary to take the first and second derivatives of the equation in order to find the required parameters. The second derivative was necessary to find the point of 
inflection. An inflection point is a point on a curve at which the curvature changes from convex to concave or vice versa. Thus, setting the second derivative equal to zero and solving for $t$ gives the point of inflection. (See Figure 2)

$$
\begin{gathered}
0=a c^{2} e^{(b-c t)-e^{(b-c t)}}\left(e^{(b-c t)}-1\right) \\
\Rightarrow t_{i}=\frac{b}{c}=6.479 .
\end{gathered}
$$

The slope of the tangent line at the point of inflection, which represents the maximum growth rate, was found using the first derivative. (See Figure 3)

$$
\text { Max growth rate: } f^{\prime}\left(t_{i}\right)=0.8018 \text {. }
$$

The lag time, $t_{L}$, is obtained by finding the $\mathrm{x}$-axis intercept of the tangent line. The slope-intercept form of a line, $y=m x+b$, was used to solve for $b$ at the point (6.4286, 2.8404). (See Figure 4)

$$
\begin{gathered}
2.8404=(0.80184) 6.4286+b \\
b=-2.3146 \\
0=0.80814 t_{L}-2.3146 \\
t_{L}=2.8867
\end{gathered}
$$

\section{Conclusions and Recommendations}

At 41.5 degrees Celsius, the growth of Lactobacillus plantarum bacteria was successfully modeled using the Gompertz curve. The asymptote, which represents the maximum relative population of the bacteria, was found to be 7.8324 . The lag time was found to be 2.8867 hours. The maximum rate of growth of Lactobacillus plantarum bacteria was found to be 0.80184 . This means that approximately eight-tenths of the relative population increased per hour during the fastest period of growth.

While the Gompertz model was a very realistic model of the growth of Lactobacillus plantarum, it was not perfect. The constants $a, b$ and $c$ that were found did give some error when compared to the experimental data. The sum of the squares of this error was approximately 0.26 . A model that is able to reduce the sum of the squared error even further would notably improve further studies of this problem.

The objective of the paper was met and the Gompertz curve was able to model the experimental data. The results have direct implications for biology and medicine because they provide the fastest rate of growth of bacteria. In addition, the results showed the asymptote and the lag time before the bacteria reaches its maximum growth rate. These results are vital to the development of products using Lactobacillus plantarum bacteria and have improved the understanding of the relationship between bacterial growth and Calculus. 


\section{References}

Banks, Robert. Growth and Diffusion Phenomena: Mathematical Frameworks and Applications. Texts in applied mathematics, 14. Berlin: Springer-Verlag, 1994.

Bielecki, Stanisław, J. Tramper, and Jacek Polak. Food Biotechnology. Progress in Biotechnology, 17. Amsterdam: Elsevier, 2000.

Niedzielin K, Kordecki H, and Birkenfeld B. "A controlled, double-blind, randomized study on the efficacy of Lactobacillus Plantarum 299V in patients with irritable bowel syndrome." Eur J Gastroenterol Hepatol. 2001 Oct;13(10):1143-7

Wessels, Stephen. "The Microorganisms We Eat - Who decides Which Ones?" Human Health and Safety. www.teknologiportalen.dk/.../Foodstuffs/QPS.htm. 27 April 2009.

Zwietering, M. H., Jongenburger, I., Rombouts, F. M., VAN 'T Riet, K.

Modeling of the Bacterial Growth Curve. Appl. Environ. Microbiol. 1990 56: 1875-1881. 


\section{Appendix}

Figure 1

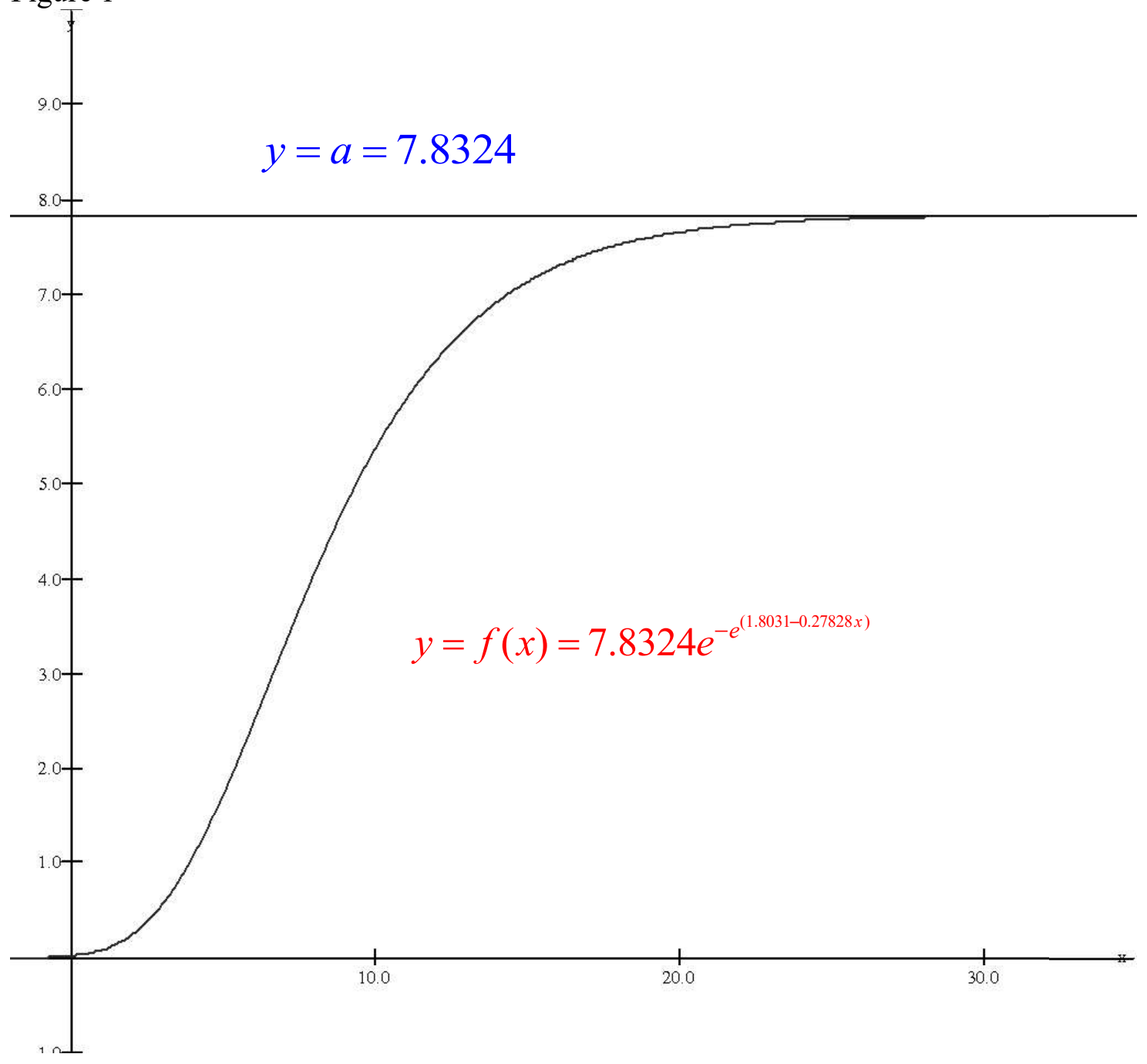




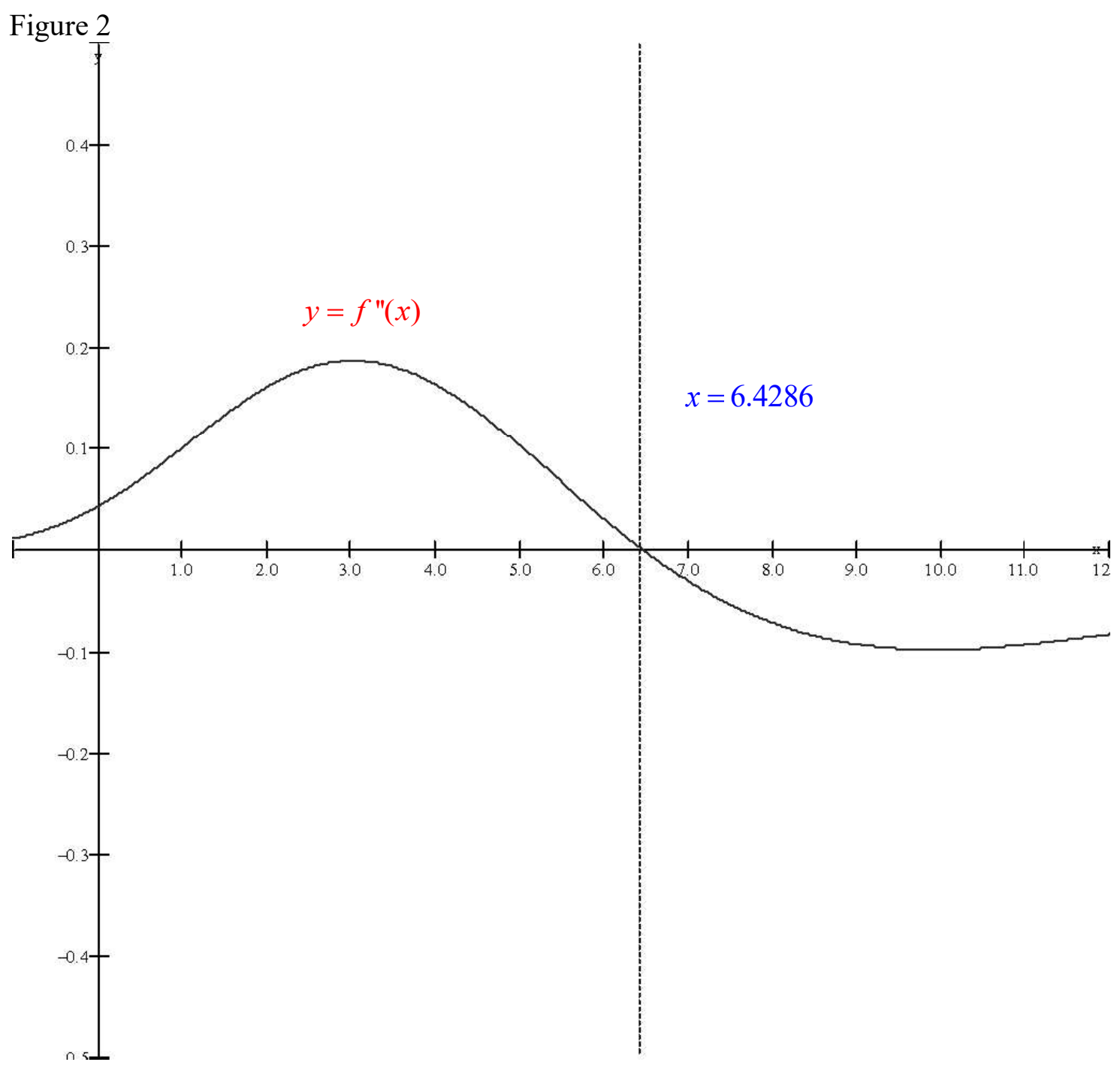


Figure 3

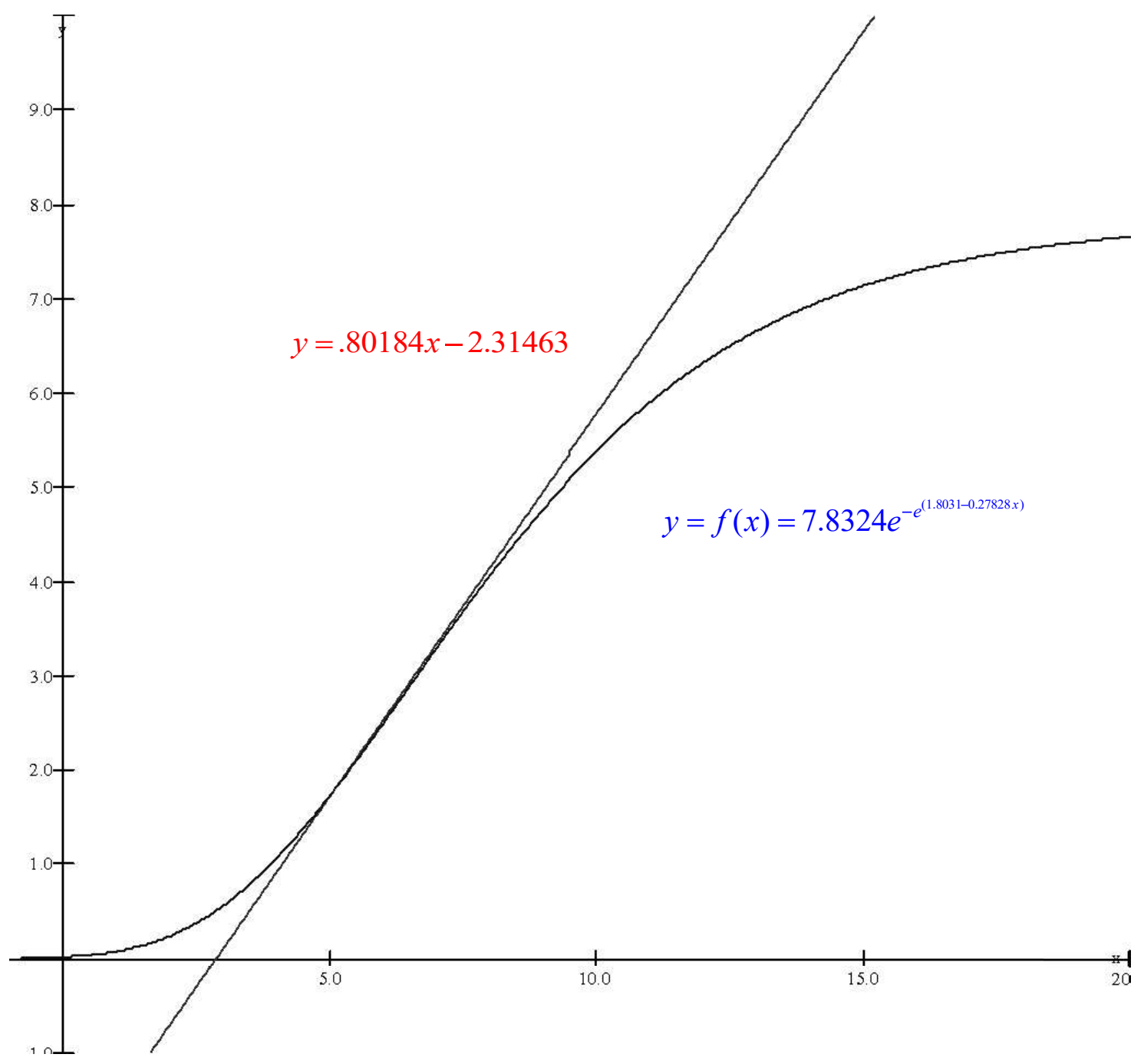


Figure 4

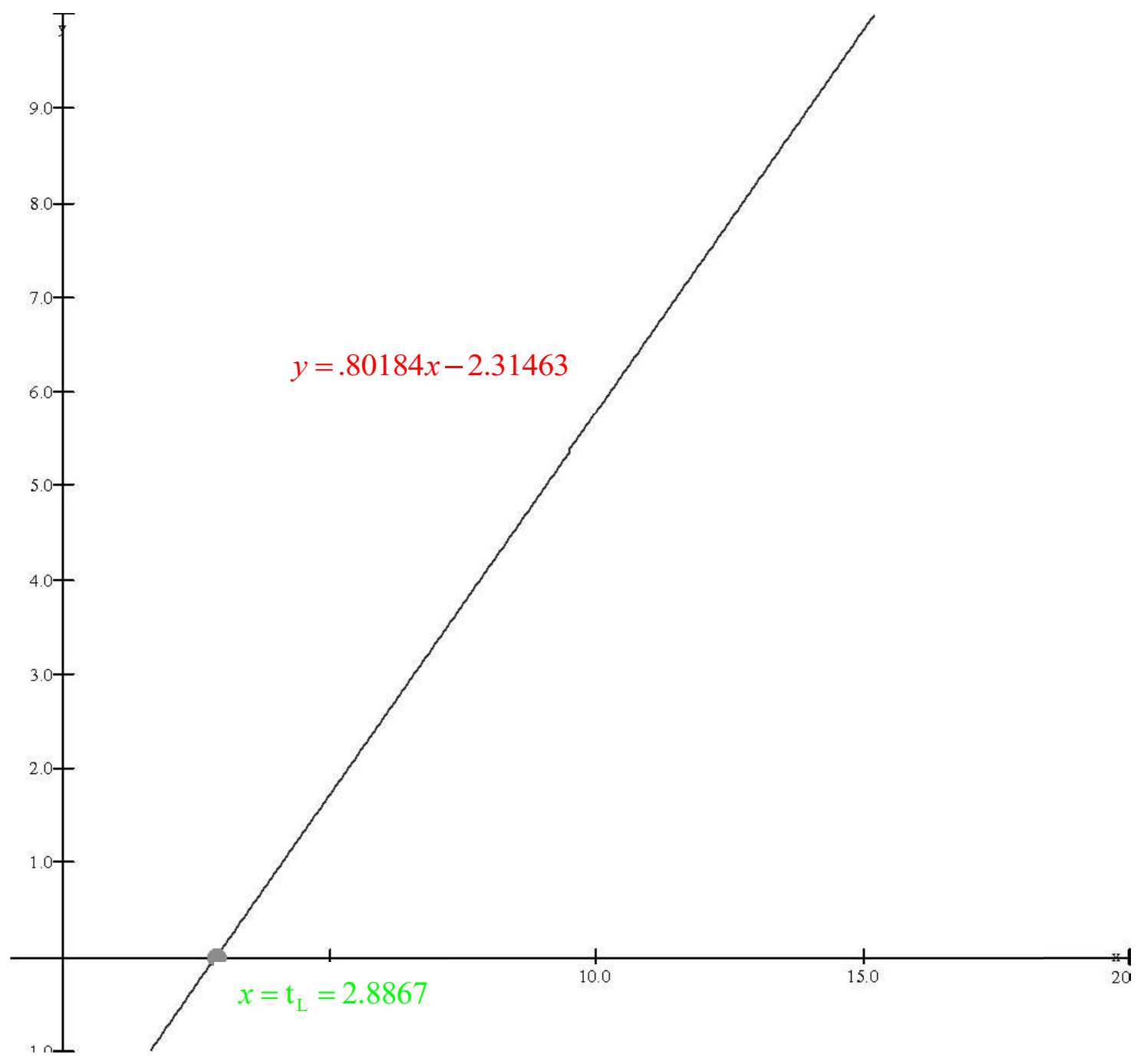

